\title{
Experiencia del departamento de hernias y pared en el manejo de endometriosis de pared abdominal durante 9 años
}

\author{
Experience of the hernia and wall department in the management of abdominal wall \\ endometriosis for 9 years
}

\author{
Alan E. Ramos-Mayo ${ }^{*}$ y Gerardo Gil-Galindo ${ }^{1,2}$

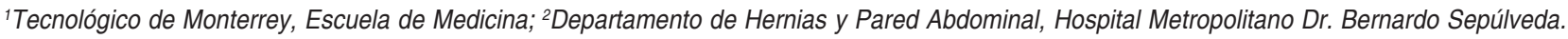 \\ Monterrey, Nuevo León, México
}

\section{Resumen}

Antecedentes: La endometriosis de pared abdominal es una enfermedad rara, se presenta como una tumoración con dolor, eritema, y que empeora a las maniobras de Valsalva. Objetivo: Mostrar la experiencia y los resultados en el manejo del departamento de hernias y pared abdominal en un hospital de segundo nivel y presentar una revisión de la literatura. Método: Se tomaron los expedientes de pacientes tratadas en el departamento de hernias y pared abdominal, intervenidas quirúrgicamente con diagnóstico clínico preoperatorio e histopatológico posoperatorio de endometriosis de pared abdominal. Los datos se describieron usando medidas de tendencia central y porcentajes. Resultados: Se identificaron 29 pacientes, con una edad promedio de 32.75 años. El 68.9\% reportó dolor cíclico catamenial asociado a tumoración de pared abdominal; se hizo diagnóstico clínico preoperatorio de endometriosis de pared abdominal en el 89.75\%. Solo una paciente se reportó con diagnóstico histopatológico de fibrolipoma. La totalidad de las pacientes refirió operación cesárea como cirugía previa. No se reportaron recurrencias. Conclusiones: La endometriosis de pared abdominal es la presencia de tejido endometrial ectópico en la pared abdominal. Las pacientes con este diagnóstico son referidas al cirujano general por presentar tumoración, sin embargo, la tasa de diagnóstico preoperatorio es baja en la mayoría de los casos. El ultrasonido es de utilidad en el diagnóstico; la tomografía y la resonancia magnética determinan la extensión de la enfermedad. El tratamiento debe relacionarse con la extensión de la enfermedad y su recurrencia se asocia con la presencia de márgenes positivos.

PALABRAS CLAVE: Endometriosis. Pared abdominal. Cicatriz quirúrgica.

\begin{abstract}
Background: Abdominal wall endometriosis is a rare pathological entity. It presents as a tumor with pain, erythema, which worsens with Valsalva maneuvers. Objective: To show the experience and results in the management of the department of hernias and abdominal wall in a hospital of second level and to present review of the literature. Method: The files of patients treated in the department of hernias and abdominal wall, surgically treated with preoperative clinical and postoperative histopathological diagnosis of abdominal wall endometriosis were taken. The data was described using measures of central tendency and percentages. Results: Twenty-nine patients were identified, with an average age of 32.75 years, $68.9 \%$ reported cyclic catamenial pain associated with a tumor in the abdominal wall; a preoperative clinical diagnosis of abdominal wall endometriosis was made in $89.75 \%$. Only one patient was reported with histopathological diagnosis of fibrolipoma. The totality of the patients referred cesarean section as previous surgery. No recurrences were reported. Conclusions: Abdominal wall endometriosis is the presence of ectopic endometrial tissue in any layer of the abdominal wall. Patients with this diagnosis are referred to the general surgeon presenting a tumor, however, the preoperative diagnosis rate is erroneous in most cases. Abdominal wall
\end{abstract}

\footnotetext{
Correspondencia:

*Alan Elison Ramos-Mayo

Av. Ignacio Morones Prieto, 3000

Col. Los Doctores

C.P. 64710 Monterrey, N.L., México

E-mail: ramosmayo.md@gmail.com
}

Fecha de recepción: 24-07-2018

Fecha de aceptación: 28-01-2019

DOI: 10.24875/CIRU.19000622

Cir Cir. 2019;87:385-389

Contents available at PubMed www.cirugiaycirujanos.com 
ultrasound is useful in diagnosis; tomography and magnetic resonance determine the extent of the disease. The treatment must be related to the extension of the disease and its recurrence is associated with the presence of positive margins.

KEY WORDS: Endometriosis. Abdominal wall. Surgical scars.

\section{Introducción}

La endometriosis es la presencia de tejido o estroma endometrial fuera del útero. El sitio más común de implantación es la pelvis, e incluye los ovarios, los ligamentos uterinos, el tabique rectovaginal y el peritoneo. Otros sitios inusuales reportados son la vejiga, el intestino, los riñones, el apéndice cecal, la cicatriz umbilical, los pulmones, el saco herniario, las extremidades y las cicatrices quirúrgicas ${ }^{1,2}$. La endometriosis de pared abdominal (EPA) es una enfermedad rara que se asocia al manejo quirúrgico del útero o de las trompas de Falopio, y se reportó por primera vez en 1956. Puede presentarse como una tumoración con dolor y eritema, que empeora con la maniobra de Valsalva. Los diagnósticos diferenciales son hematoma, seroma, granuloma, absceso, tumor, hernia incisional, tumor desmoide, sarcoma, linfoma y cáncer primario o metastásico ${ }^{2,3}$. El objetivo de este estudio es presentar la experiencia y los resultados en el manejo por parte del departamento de hernias y pared abdominal en un hospital de segundo nivel, y hacer una revisión de la literatura.

\section{Método}

Se tomaron los expedientes de pacientes tratadas en el departamento de hernias y pared abdominal, que hubieran sido intervenidas quirúrgicamente con diagnóstico clínico preoperatorio e histopatológico posoperatorio de EPA. Las variables que se incluyeron fueron edad, tiempo de evolución con la enfermedad, dolor cíclico catamenial, tumor primario o recurrente, diagnóstico clínico preoperatorio, diagnóstico preoperatorio por estudios de imagen, tamaño de la tumoración, tipo de manejo quirúrgico realizado, diagnóstico histopatológico y tasa de recurrencia.

En el análisis estadístico los datos se describieron usando medidas de tendencia central y porcentajes.

\section{Resultados}

Se analizaron registros hospitalarios desde enero de 2008 a diciembre de 2017. Se reportó un total de 29 pacientes, que se incluyeron en este análisis. La edad promedio de las pacientes fue 32.75 años (rango: 24-47 años), y el tiempo promedio de evolución con la enfermedad fue de 30.2 meses (rango: 8-60 meses). La totalidad de las pacientes referían tumoración palpable, $20(68.9 \%)$ refirieron dolor cíclico catamenial y $9(20.7 \%)$ negaron la presencia de dolor. Los diagnósticos clínicos preoperatorios fueron EPA en 26 pacientes (89.75\%), fibroma de pared abdominal en una (3.45\%), lipoma de pared abdominal en otra $(3.45 \%)$, y una más con hernia ventral incisional (3.45\%); en estas tres pacientes (10.35\%) se confirmó el diagnóstico de EPA por reporte histopatológico posoperatorio (Figs. 1 y 2). En una de las pacientes con diagnóstico preoperatorio de EPA se hizo diagnóstico histopatológico posoperatorio de fibrolipoma. El $96.6 \%$ de las pacientes presentaban tumoración primaria y antecedente de operación cesárea. Se realizó ultrasonido preoperatorio en 14 pacientes (48.2\%); las otras $15(51.8 \%)$ no tuvieron estudios de imagen (Tabla 1).

El tamaño promedio del tumor fue de $13.48 \mathrm{~cm}^{2}$ (rango: 2-35 $\left.\mathrm{cm}^{2}\right)$. Dos pacientes $(6.9 \%)$ recibieron manejo médico con danazol, sin mejoría de los síntomas. En una paciente había sospecha de endometriosis pélvica, pero no se confirmó y tuvo resolución de los síntomas después de la cirugía. Los manejos quirúrgicos realizados fueron (Fig. 3): escisión de la tumoración con cierre primario de la aponeurosis en 17 pacientes $(58.62 \%)$, escisión del tumor con procedimiento de Rives y malla de polipropileno en 9 pacientes (31.03\%), escisión del tumor en tejido celular subcutáneo en una paciente (3.45\%), escisión del tumor con colocación de malla de polipropileno onlay en una paciente (3.45\%), y escisión del tumor con procedimiento de Lichtenstein en otra paciente $(3.45 \%)$ por extensión de tumor a la región inguinal. Al momento del estudio no se habían reportado recurrencias (Tabla 2 ).

\section{Discusión}

La endometriosis es una enfermedad común, benigna, dependiente de estrógenos, que se asocia a dolor pélvico e infertilidad. Se caracteriza por la presencia de tejido endometrial fuera del útero ${ }^{1,4}$. Afecta al 6-10\% de las mujeres en edad reproductiva, y está presente 


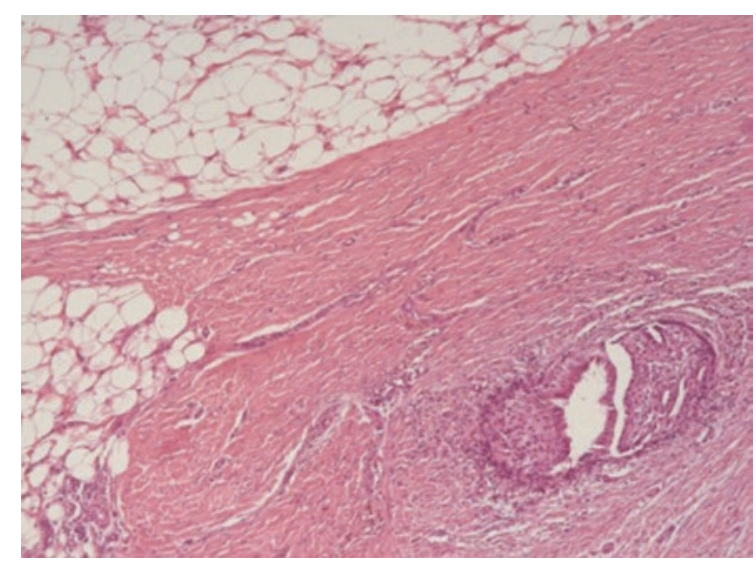

Figura 1. Tejido glandular endometrial rodeado de tejido adiposo.

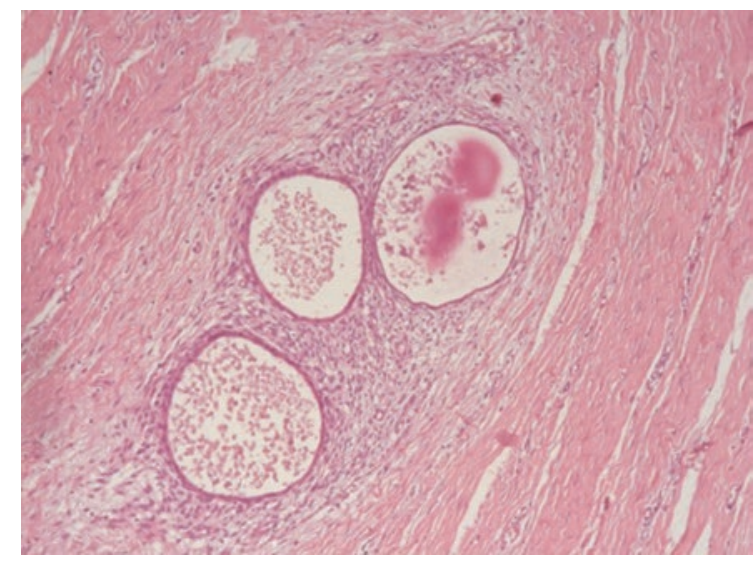

Figura 2. Tejido glandular endometrial.

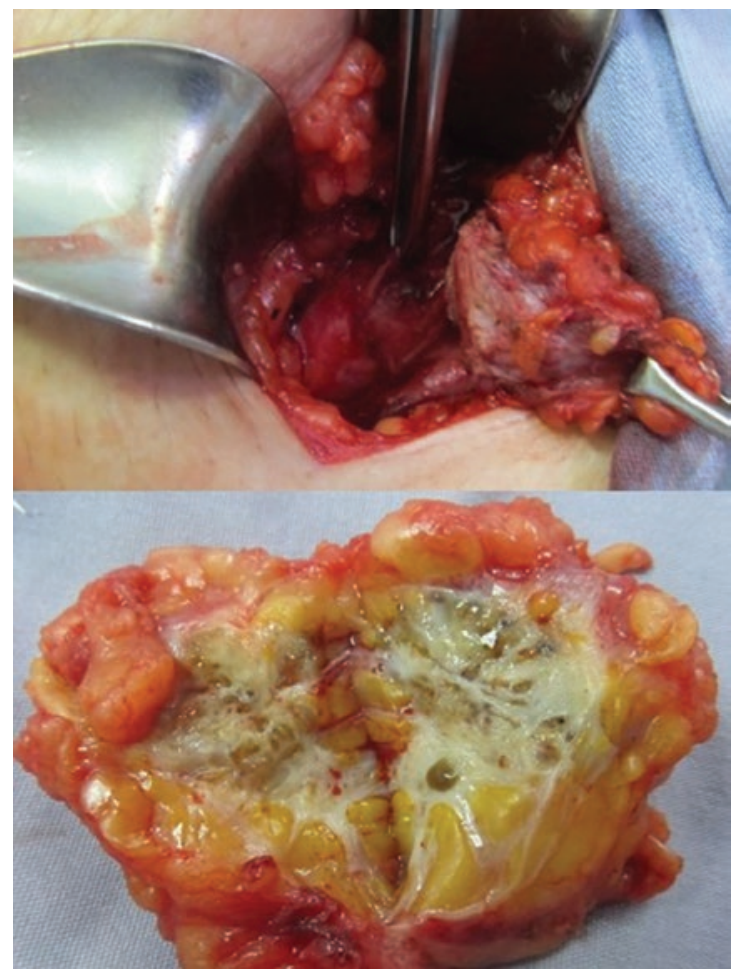

Figura 3. Escisión de endometrioma de pared abdominal.
Tabla 1. Datos demográficos

\begin{tabular}{|c|c|c|c|c|}
\hline & $\mathbf{n}$ & $\%$ & Media & Rango \\
\hline Edad & 29 & 100 & 32.75 años & 27-47 años \\
\hline Tiempo de evolución & & & 30.2 meses & 8-60 meses \\
\hline \multicolumn{5}{|l|}{ Hallazgos clínicos } \\
\hline Tumoración & 29 & 100 & & \\
\hline Dolor cíclico & 20 & 68.9 & & \\
\hline Cirugía previa cesárea & 29 & 100 & & \\
\hline \multicolumn{5}{|c|}{ Diagnóstico preoperatorio } \\
\hline $\begin{array}{l}\text { Endometriosis de pare } \\
\text { abdominal }\end{array}$ & 26 & 89.7 & & \\
\hline Fibroma & 1 & 3.44 & & \\
\hline Lipoma & 1 & 3.44 & & \\
\hline Hernia & 1 & 3.44 & & \\
\hline \multicolumn{5}{|l|}{ Características del tumor } \\
\hline Primario & 28 & 96.6 & & \\
\hline Recurrente & 1 & 3.44 & & \\
\hline \multicolumn{5}{|c|}{ Ultrasonografía preoperatoria } \\
\hline Sí & 14 & 48.2 & & \\
\hline No & 15 & 51.8 & & \\
\hline
\end{tabular}

Tabla 2. Datos clínicos

\begin{tabular}{|c|c|c|c|c|}
\hline Variable & $\mathrm{n}$ & $\%$ & Media & Rango \\
\hline Área de la tumoración & & & $13.48 \mathrm{~cm}^{2}$ & $2-35 \mathrm{~cm}^{2}$ \\
\hline \multicolumn{5}{|l|}{ Tratamiento médico } \\
\hline Sí & 2 & 6.9 & & \\
\hline No & 27 & 93.1 & & \\
\hline \multicolumn{5}{|l|}{ Tratamiento quirúrgico } \\
\hline Resección & 1 & 3.44 & & \\
\hline $\begin{array}{l}\text { Resección y cierre primario } \\
\text { aponeurosis }\end{array}$ & 17 & 58.62 & & \\
\hline Resección y plastia tipo Rives & 9 & 31.02 & & \\
\hline Resección y plastia onlay & 1 & 3.44 & & \\
\hline Resección y plastia Lichtenstein & 1 & 3.44 & & \\
\hline \multicolumn{5}{|l|}{ Diagnóstico definitivo } \\
\hline $\begin{array}{l}\text { Endometriosis de pared } \\
\text { abdominal }\end{array}$ & 28 & 96.6 & & \\
\hline Fibrolipoma & 1 & 3.44 & & \\
\hline \multicolumn{5}{|l|}{ Recurrencia } \\
\hline No & 29 & 100 & & \\
\hline
\end{tabular}

en el $35-50 \%$ de las pacientes que presentan dolor pélvico, infertilidad o ambos ${ }^{4,5}$. La teoría más aceptada sobre la patogénesis de esta enfermedad fue propuesta en 1920 y concluye que se debe a una menstruación retrógrada a la cavidad uterina a través de las trompas de Falopio, y que conlleva la implantación de tejido endometrial en sitios pélvicos o extrapélvicos. La segunda teoría más aceptada es que acontece por diseminación vascular, por células endometriales que escapan del útero a través de canales linfovasculares 
y tienen acceso a la circulación periférica, lo que implanta este tejido en sitios ectópicos distales. Y una tercera teoría refiere que hay metaplasia de las células pluripotenciales de la pared abdominal, que cambian a tejido endometrial, y este proceso está inducido por manipulación hormonal y puede presentarse principalmente en la adolescencia ${ }^{4,6,7}$.

La EPA es la presencia de tejido endometrial ectópico en la superficie del peritoneo, la aponeurosis o el tejido celular subcutáneo. La mayor incidencia se asocia a la operación cesárea, y el mecanismo secundario se cree que es la implantación de tejido endometrial en la cicatriz quirúrgica ${ }^{1,2,8}$. Esta enfermedad se reporta por primera vez en 1956 con casos anecdóticos en la literatura ginecológica y hasta 1975 en la literatura de cirugía general. Su incidencia es variable: después de una operación cesárea algunos estudios reportan una incidencia del $0.03-1 \%$ y en algunas series llega hasta el 3.5\%; tras histerectomías por aborto del segundo trimestre se reportan incidencias del 1.08\%,1,2,910. Las manifestaciones clínicas pueden aparecer desde 6 meses hasta 10 años después de la cirugía previa, y destacan la presencia de tumoración en la pared abdominal con aumento gradual de tamaño en el $96 \%$ de los casos y dolor cíclico catamenial con tumoración en el $57 \%$ de los casos; algunos autores sugieren esta manifestación como dato patognomónico de la enfermedad, pero también puede existir tumoración sin asociación a dolor cíclico, que se puede presentar en el $87 \%$ de los $\operatorname{casos}^{2,6,7,11}$.

Las pacientes con EPA son referidas al cirujano general por la presencia de un tumor de pared abdominal; sin embargo, la tasa de diagnóstico preoperatorio es errónea en la mayoría de los casos, y no se obtiene un diagnóstico definitivo hasta tener un estudio histopatológico. El diagnóstico más común con el que se envían es el de hernia incisional ${ }^{2,3,12}$. El primer reporte de esta patología por cirugía general se registra en 1975, y la revisión más grande presenta 445 pacientes de diferentes estudios ${ }^{1,7}$. Las tasas de diagnóstico preoperatorio varían entre el 0 y el $42 \%, 6,11,13,14$.

Durante la exploración física se espera documentar la presencia de tumoración y determinar la extensión de la enfermedad, si hay tumor único o múltiples, y si se involucra tejido celular subcutáneo, aponeurosis, músculo, peritoneo o todas las capas de la pared abdominal, para posteriormente definir el abordaje quirúrgico ${ }^{7,10}$. Algunos autores sugieren la presencia de tumoración y de dolor cíclico como indicativa de cirugía, y otros sugieren el uso de apoyo diagnóstico para confirmar la enfermedad y planear el abordaje quirúrgico. Los hallazgos en el ultrasonido de pared abdominal son lesión quística, poliquística, mixta o sólida en el tejido celular subcutáneo, el tejido muscular, la aponeurosis o infiltrando todos los tejidos; en el Doppler, el patrón ecográfico más común es un nódulo redondeado hipoecoico con cambios fibróticos, anillo periférico hiperecoico, márgenes espiculados y pedículo vascular único que puede o no relacionarse con los ciclos menstruales y que se presenta en el $74 \%$ de los casos; otros hallazgos menos comunes son estructura heterogénea, presencia de pequeñas áreas quísticas dentro de la lesión, tracto fistuloso que comunica a la piel o al músculo, y múltiples pedículos vasculares $7,10,11,15,16$. Los hallazgos en la tomografía computada pueden ser una masa sólida o mixta bien circunscrita, con hemorragia o no, que puede ser isodensa comparada con el tejido muscular y que realza al contraste. Los hallazgos en la resonancia magnética son similares a los observados en la endometriosis pélvica y con señales de alta intensidad en T1 y T2 consistentes con hemorragia subaguda. En general, los hallazgos pueden ser inespecíficos en ambos estudios, y la utilidad de la tomografía y de la resonancia magnética reside en determinar la extensión de la enfermedad $2,17-19$.

Otra opción en el abordaje diagnóstico de la EPA es la biopsia por aspiración. Se obtiene una muestra para frotis, en el que se demostrarán fragmentos epiteliales y estromales, con características histológicas de endometriosis. Este método ofrece certeza diagnóstica, pero los estudios de imagen, en conjunto con el cuadro clínico, lo hacen con similar eficacia y sin el riesgo invasivo ${ }^{20-22}$. El diagnóstico definitivo está determinado por el estudio histopatológico de la muestra o del tejido extraído, y generalmente el diagnóstico preoperatorio de EPA está ausente ${ }^{6,20,22}$.

El tratamiento se relaciona con la extensión de la enfermedad, y cuando se asocie a enfermedad pélvica se tomarán en cuenta los deseos de la paciente para mantener su capacidad reproductiva. Se ha propuesto manejo con vigilancia, manejo médico basado en anticonceptivos orales y agonistas de la hormona liberadora de gonadotropina, y tratamiento quirúrgico con escisión amplia con márgenes libres de tumor de hasta $1 \mathrm{~cm}$, siendo este último tratamiento el preferido para el manejo de las lesiones de la pared abdominal por su bajo riesgo de recurrencia y resolución de la sintomatología ${ }^{2,13,22}$. Debe considerarse la resección en bloque de la lesión que incluya elementos miofasciales de ser necesario, y hay que estar preparado para el manejo de patología herniaria coexistente e incluso la colocación de una malla. La tasa de recurrencias se estima en un $3-4.3 \%$ y se asocia a 
márgenes positivos presentes en la resección inicial; se sugiere que un diagnóstico preoperatorio adecuado condicionará un tratamiento óptimo $0^{1,7,12}$.

Podemos concluir que la EPA es una enfermedad de baja incidencia, con comportamiento incierto y que requiere un alto índice de sospecha para realizar un diagnóstico clínico preoperatorio adecuado. El abordaje diagnóstico puede precisar ocasionalmente estudios de imagen con el objetivo de delimitar la extensión de la enfermedad y planear un tratamiento adecuado, con márgenes libres de tumoración. Su alta asociación con la operación cesárea sugiere que puede ser una patología potencialmente prevenible, y debe sospecharse este diagnóstico en toda paciente con tumoración de pared abdominal y antecedente de operación cesárea. La valoración realizada por un cirujano general con alto índice se sospecha sobre esta enfermedad se asocia a mejores tasas de diagnóstico preoperatorio. Las tasas de recurrencia vienen determinadas por la efectividad en lograr una resección libre de lesión.

\section{Financiamiento}

No se recibió ninguna beca ni apoyo para la realización de este estudio.

\section{Conflicto de intereses}

Los autores declaran que no hay conflicto de intereses.

\section{Agradecimientos}

Agradecemos al Departamento de Hernias y Pared Abdominal del Hospital Metropolitano Dr. Bernardo Sepúlveda por facilitar la realización de este estudio.

\section{Responsabilidades éticas}

Protección de personas y animales. Los autores declaran que para esta investigación no se han realizado experimentos en seres humanos ni en animales.
Confidencialidad de los datos. Los autores declaran que han seguido los protocolos de su centro de trabajo sobre la publicación de datos de pacientes.

Derecho a la privacidad y consentimiento informado. Los autores han obtenido el consentimiento informado de los pacientes y/o sujetos referidos en el artículo. Este documento obra en poder del autor de correspondencia.

\section{Bibliografía}

1. Seydel AS, Sickel JZ, Warner ED, Sax HC. Extrapelvic endometriosis: diagnosis and treatment. Am J Surg. 1996;171:239.

2. Blanco RG, Parithivel VS, Shah AK, Gumbs MA, Schein M, Gerst PH. Abdominal wall endometriomas. Am J Surg. 2003;185:596-8.

3. Wolf $\mathrm{Y}$, Haddad R, Werbin N, Skornick Y, Kaplan O. Endometriosis in abdominal scars: a diagnostic pitfall. Am Surg. 1996;62:1042-4.

4. Giudice LC, Kao LC. Endometriosis. Lancet. 2004;364:1789-99.

5. Giudice LC. Clinical practice. Endometriosis. N Engl J Med. 2010; 362:2389-98.

6. Col C, Yilmaz EE. Cesarean scar endometrioma: case series. World $J$ Clin Cases. 2014;2:133-6.

7. Horton JD, Dezee KJ, Ahnfeldt EP, Wagner M. Abdominal wall endometriosis: a surgeon's perspective and review of 445 cases. Am J Surg. 2008;196:207-12.

8. Brenner C, Wohlgemuth S. Scar endometriosis. Surg Gynecol Obstet. 1990;170:538-40.

9. Honore GM. Extrapelvic endometriosis. Clin Obstet Gynecol. 1999; 42:699-711.

10. Nirula R, Greaney GC. Incisional endometriosis: an underappreciated diagnosis in general surgery. J Am Coll Surg. 2000;190:404-7.

11. Patterson GK, Winburn GB. Abdominal wall endometriomas: report of eight cases. Am Surg. 1999;65:36-9.

12. Khamechian T, Alizargar J, Mazoochi T. 5-Year data analysis of patients following abdominal wall endometrioma surgery. BMC Womens Health. 2014;14:151.

13. Firilas A, Soi A, Max M. Abdominal incision endometriomas. Am Surg. 1994:60:259-61.

14. Singh KK, Lessells AM, Adam DJ, Jordan C, Miles WF, Macintyre IM, et al. Presentation of endometriosis to general surgeons: a 10-year experience. Br J Surg. 1995;82:1349-51.

15. Francica G. Reliable clinical and sonographic findings in the diagnosis of abdominal wall endometriosis near cesarean section scar. World $\mathrm{J}$ Radiol. 2012;4:135-40.

16. Woodward PJ, Sohaey R, Mezzetti TP, Jr. Endometriosis: radiologic-pathologic correlation. Radiographics. 2001;21:193-216; questionnaire 88-94.

17. Amato $M$, Levitt R. Abdominal wall endometrioma: $C T$ findings. J Comput Assist Tomogr. 1984;8:1213-4.

18. Hensen JH, Van Breda Vriesman AC, Puylaert JB. Abdominal wall endometriosis: clinical presentation and imaging features with emphasis on sonography. AJR Am J Roentgenol. 2006;186:616-20.

19. Yu CY, Perez-Reyes M, Brown JJ, Borrello JA. MR appearance of umbilical endometriosis. J Comput Assist Tomogr. 1994;18:269-71.

20. Pachori G, Sharma R, Sunaria RK, Bayla T. Scar endometriosis: diagnosis by fine needle aspiration. J Cytol. 2015;32:65-7.

21. Gupta RK, Green C, Wood KP. Fine needle aspiration cytodiagnosis of endometriosis in an abdominal scar after caesarean section. Cytopathology. 2000;11:67-8.

22. Machairiotis N, Stylianaki A, Dryllis G, Zarogoulidis P, Kouroutou P, Tsiamis $\mathrm{N}$, et al. Extrapelvic endometriosis: a rare entity or an under diagnosed condition? Diagn Pathol. 2013;8:194. 